\title{
Surgical Treatment of a Parastomal Hernia
}

\author{
Seung Chul Heo, Heung-Kwon $\mathrm{Oh}^{1}$, Yoon Suk Song ${ }^{1}$, Mi Sun Seo ${ }^{1}$, Eun Kyung Choe ${ }^{2}$, Seungbum Ryoo ${ }^{3}$, \\ Kyu Joo Park ${ }^{1}$ \\ Department of Surgery, Seoul Metropolitan Government Seoul National University Boramae Medical Center, Seoul; ${ }^{1}$ Department of Surgery, \\ Seoul National University Hospital, Seoul; ${ }^{2}$ Seoul National University Hospital Health Care System Kangnam Center, Seoul; ${ }^{3}$ Department of \\ Surgery, Dongnam Institute of Radiological and Medical Sciences, Busan, Korea
}

\begin{abstract}
Purpose: Parastomal hernia is a major complication of an intestinal stoma. This study was performed to compare the results of various operative methods to treat parastomal hernias.

Methods: Results of surgical treatment for parastomal hernias (postoperative recurrence, complications and postoperative hospital stays) were surveyed in 39 patients over an 11-year period. The patients enrolled in this study underwent surgery by a single surgeon to exclude surgeon bias.

Results: Seventeen patients were male, and twenty-two patients were female. The mean age was 65.9 years (range, 36 to 86 years). The stomas were 35 sigmoid-end-colostomies (90\%), 2 loop-colostomies (5\%), and 2 double-barrel-colostomies. Over half of the hernias developed within two years after initial formation. Stoma relocation was performed in 8 patients, suture repair in 14 patients and mesh repair in 17 patients. Seven patients had recurrence of the hernia, and ten patients suffered from complications. Postoperative complications and recurrence were more frequent in stoma relocation than in suture repair and mesh repair. Emergency operations were performed in four patients (10.3\%) with higher incidence of complications but not with increased risk of recurrence. Excluding emergency operations, complications of relocations were not higher than those of mesh repairs. Postoperative hospital stays were shortest in mesh repair patients.

Conclusion: In this study, mesh repair showed low recurrence and a low complication rate with shorter hospital stay than relocation methods, though these differences were not statistically significant. Further studies, including randomized trials, are necessary if more reliable data on the surgical treatment of parastomal hernias are to be obtained.
\end{abstract}

\section{Keywords: Parastomal hernia; Recurrence; Complication; Relocation; Mesh repair}

\section{INTRODUCTION}

A variety of complications is associated with stomas. Skin-related complications, bowel obstructions, stoma retractions and parastomal hernias are frequent in ileosotmies [1]; parastomal hernias, skin complications and prolapses are frequent complications for colostomies [2]. Parastomal hernias develop in 5-30\% of stoma patients and cause asymmetries of the abdominal wall and difficulties in

Received: April 14, 2011 Accepted: August 3, 2011

Correspondence to: Kyu Joo Park, M.D.

Department of Surgery, Seoul National University College of Medicine,

28 Yeongeon-dong, Jongno-gu, Seoul 110-744, Korea

Tel: +82-2-2072-2901, Fax: +82-2-766-3975

E-mail: kjparkmd@plaza.snu.ac.kr

(C) 2011 The Korean Society of Coloproctology

This is an open-access article distributed under the terms of the Creative Commons Attribution NonCommercial License (http://creativecommons.org/licenses/by-nc/3.0) which permits unrestricted noncommercial use, distribution, and reproduction in any medium, provided the original work is properly cited. applying stoma bags. Even though poor general conditions of some patients prohibit surgical corrections, aggressive treatments are necessary for patients with pain, problems in wearing stoma appliances, prolapses of stomas, necrosis of a herniated bowel and serious cosmetic problems [3]. Suggested causes of parastomal hernias are postoperative weight gain, increased intra-abdominal pressure combined with weakened abdominal muscles, and old age; other than these, hernias can occur in cases of excessive extension or contraction of a muscle and in cases of improper parastomal anchoring [4].

Correction of a parastomal hernia is not easy, and high recurrence rates after surgical corrections are reported [5]. Relocation of the stoma has been known to cause a low recurrence rate in the treatment of parastomal hernias, but recently local correction, including primary closure of the fascial defects with reinforcement by artificial membrane, has been preferred, and prevention of a hernia by using artificial membranes have frequently been tried. In this study, by analyzing the results for the each surgical method, 
we attempted to find a suitable surgical method for repairing a parastomal hernia.

\section{METHODS}

The 39 patients who underwent surgery for parastomal hernias in the Department of Surgery, Seoul National University Hospital, from June 1998 to December 2009 were enrolled in this study. Patients operated on by a single surgeon were included in order to exclude surgeon bias. The types and the locations of the initial stomas, the surgical methods for repairing the parastomal hernias and the results of surgery were surveyed retrospectively based on the medical records. For the surgical results, we compared recurrence rate, complication rates, and postoperative hospital stays. During the study period, another 13 patients with parastomal hernias of the ileal conduit underwent surgery for parastomal hernias; however, due to the special nature of the ileal conduit, relocation was not feasible. These cases were excluded from our study because the surgical method was one of the items being compared.

\section{RESULTS}

\section{Characteristics of patients}

During the study period, there were 17 male patients and 22 female patients who underwent surgery for parastomal hernias; their mean age was $65.9 \pm 11.6$ years. Among the preexisting stomas, end colostomies were the most, and loop colostomies were next, with double-barrel colostomies bring the least. Among the patients, one had both an end colostomy and ileal conduit, and the parastomal hernia occurred around the colostomy (Table 1).

Table 1. Demographics of the patients

\begin{tabular}{lrcr}
\hline & Male & Female & Total \\
\hline End sigmoidostomy & 15 & 20 & 35 \\
Loop colostomy & 1 & 1 & 2 \\
Double barrel ileocolostomy & 1 & 1 & 2 \\
Total & 17 & 22 & 39 \\
\hline
\end{tabular}

The original diseases causing the stomas were malignant tumors, such as rectal cancer, ovarian cancer, and bladder cancer, in 33 patients and benign conditions, such as abdominal trauma (from car accident), rectal perforation, and colonic diverticulitis, in 6 patients. The mean interval from the stoma formation to the diagnosis of a parastomal hernia was 5 years; the diagnosis rate was high during the early postoperative period, and over $50 \%$ of the patients had a hernia occurring within 2 years after stoma formation (Fig. 1).

\section{Surgical methods and results}

Three types of surgical operations were performed to repair the parastomal hernias. Relocation of the stoma was done by making a new stoma under laparotomy at a different site, with closure of the original stoma (8 cases); suture repair was performed by closing the fascial defect beneath the skin flap with interrupt sutures (14 cases); mesh repair was done by reinforcement with prolene mesh after suture repair of the fascial defects (17 cases) (Table 2). Relocation was the major surgical method in the early part of the study period, and mesh repair was the major surgical method in the later part of the study period (Fig. 2).

There were 10 postoperative complications, such as wound infec-

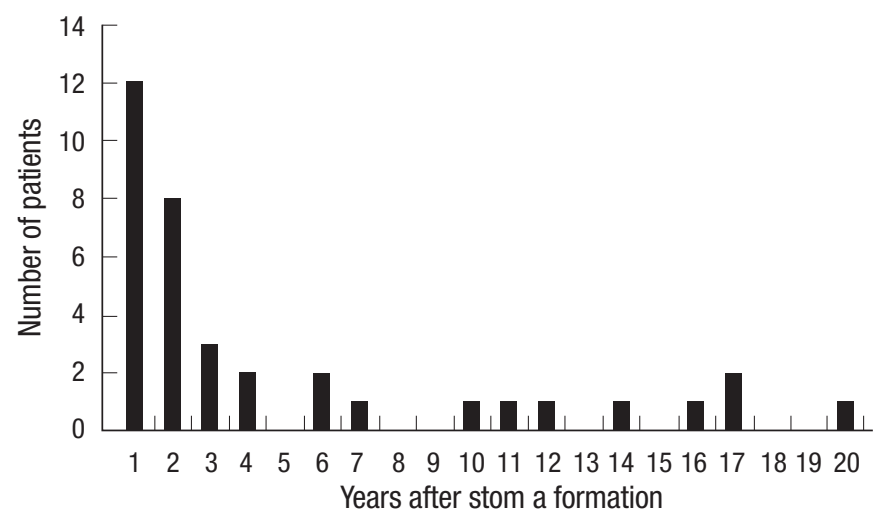

Fig. 1. Development of a parastomal hernia after stoma formation. Over half of the parastomal hernias develop during the first two years after stoma formation. Three patients with obscure dates of stoma formation were excluded from this chart.

Table 2. Results of surgery according to the operative method

\begin{tabular}{|c|c|c|c|c|c|}
\hline & No. of patients & Complications (\%) & Recurrence (\%) & $\begin{array}{c}\text { Postoperative } \\
\text { hospital stay (day) }{ }^{a}\end{array}$ & $\begin{array}{c}\text { Cases of } \\
\text { emergency operation }\end{array}$ \\
\hline Relocation & 8 & $3(37.5)$ & $3^{b}(37.5)$ & 24.8 & 2 \\
\hline Suture repair & 14 & $4(28.6)$ & $2^{c}(14.3)$ & 9.7 & 2 \\
\hline Mesh repair & 17 & $3(17.6)^{d}$ & $2^{e}(11.8)^{d}$ & $4.8^{f}$ & 0 \\
\hline Total & 39 & $10(25.6)$ & $7(17.9)$ & & 4 \\
\hline
\end{tabular}

${ }^{a}$ No difference between relocation and suture repair (Mann-Whitney $U$ test). ${ }^{\mathrm{b}}$ Two of the three patients underwent suture repair, and the other underwent mesh repair afterwards. However, one of the two patients with suture repair had recurrence again. ' One patient underwent suture repair, and the other underwent mesh repair afterwards. ${ }^{\mathrm{d} P}>0.05$ to other methods (Fisher's exact test). ${ }^{\mathrm{e}}$ These patients underwent suture repair afterwards. ${ }^{\mathrm{f}} \mathrm{P}=0.001$ to relocation method and $\mathrm{P}=0.029$ to suture repair method (Mann-Whitney U-test). 
tions, ileus, voiding difficulty, pneumonia, etc. Postoperative complications were more frequent in relocation and suture repair (Table 3). Among the initial operations for hernia repair, the parastomal hernia recurred in seven patients. The recurrence rate was highest in relocation patients while the recurrence rates in suture repair and in mesh repair were similar (Table 2), but the difference was not statistically significant. The postoperative follow-up period was $52.6 \pm 42.3$ months in relocation, $30.1 \pm 26.6$ months in suture repair and 29.6 \pm 19.6 in mesh repair patients.

For five of the seven recurrent patients, suture repairs were performed again, and for the other two patients mesh repairs were performed. Among the seven patients, one of the suture repair patients had a recurrence again, and relocation was done again. Therefore, there were 8 recurrences in 7 of the 39 patients who underwent surgery for parastomal hernias, and forty-seven operations for parastomal hernias were performed altogether (Table 4, Fig. 3)

Postoperative hospital stay was shortest for the mesh repair patients $(\mathrm{P}<0.05$, Mann-Whitney U-test).

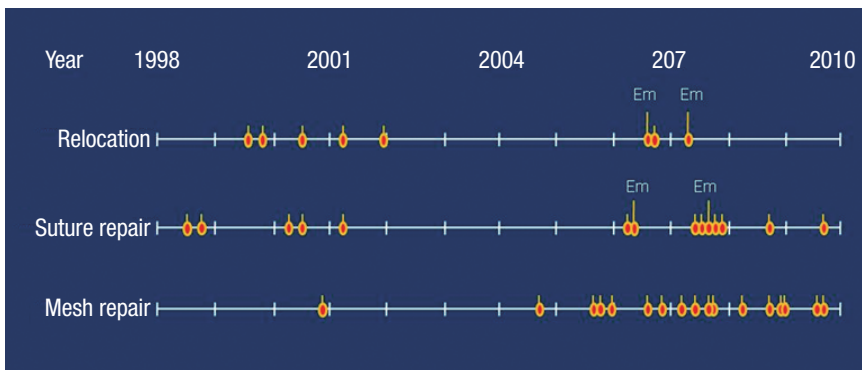

Fig. 2. Time point of each operation. Most of the relocation operations were performed in the early period while most of the mesh repairs were performed in the late period. Each ellipse indicates an operative time point. An ellipse with a long bar indicates an emergency operation.

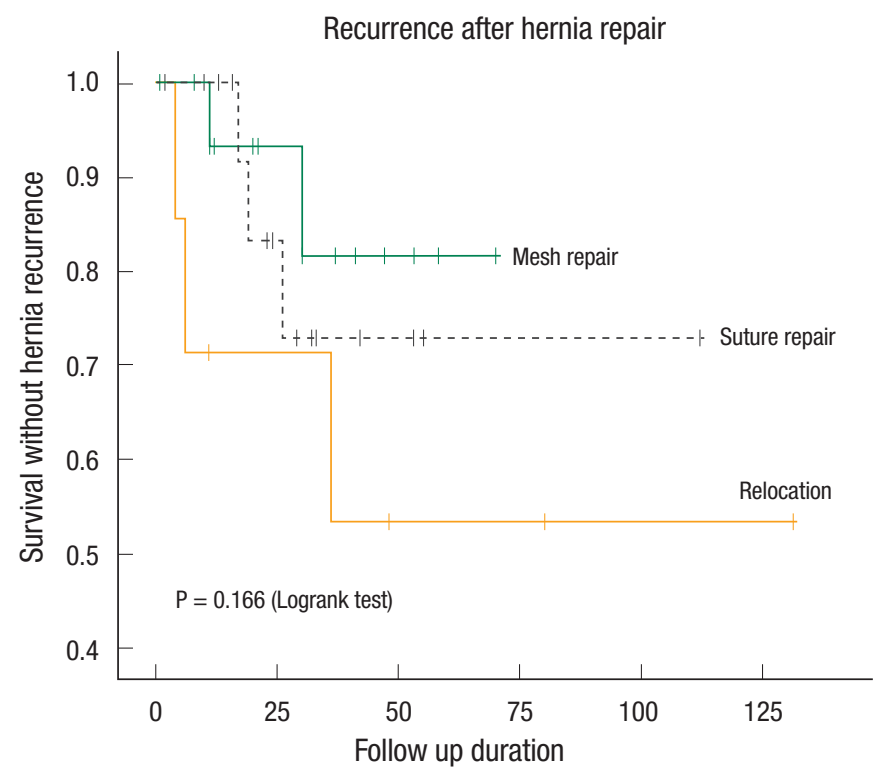

Fig. 3. Recurrence of the hernia according to the operative method. The recurrence curves for each method look separate, but the differences are not statistically significant. The total number of operations is forty-seven.

Table 4. Recurrence for each method in the total operations

\begin{tabular}{lcc}
\hline & No. of operations & Recurrence $^{\mathrm{a}}(\%)$ \\
\hline Relocation & $9(8+1)$ & $3(33.3)$ \\
Suture repair & $19(14+5)$ & $3(15.8)$ \\
Mesh repair & $19(17+2)$ & $2(10.5)$ \\
Total & 47 & $8(17.0)$ \\
\hline
\end{tabular}

ap $>0.05$ between any two methods (Fisher's exact test).

Table 3. Postoperative complications

\begin{tabular}{lcccccc}
\hline & Wound infection & lleus & Urinary retention & Pneumonia & $\begin{array}{c}\text { Multiple } \\
\text { complications }\end{array}$ & No. (\%) \\
\hline Relocation $(n=8)$ & $1^{\text {a }}$ & 1 & 0 & 0 & $1^{\text {a }}$ & $3(37.5)$ \\
Suture repair $(n=14)$ & 1 & 1 & 1 & $1^{\text {a }}$ & 0 & $4(28.6)$ \\
Mesh repair $(n=17)$ & 0 & 2 & 1 & 0 & 0 & $3(17.6)$ \\
Total $(n=39)$ & 2 & 4 & 2 & 1 & 1 & $10(25.6)$ \\
\hline
\end{tabular}

Each numeric represents the number of patients, and there was no patient with two or more complications.

${ }^{\text {aE} E m e r g e n c y ~ o p e r a t i o n ~ c a s e . ~}$

Table 5. Recurrences and complications in elective or emergency operations

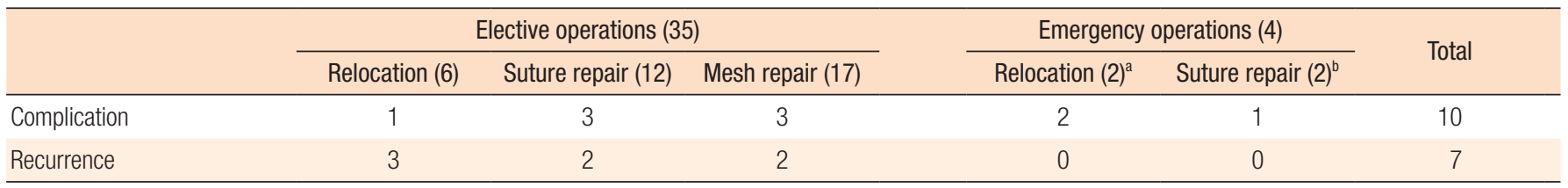

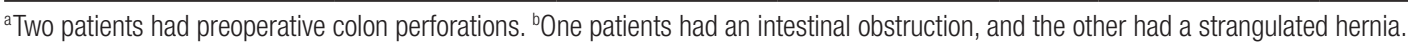



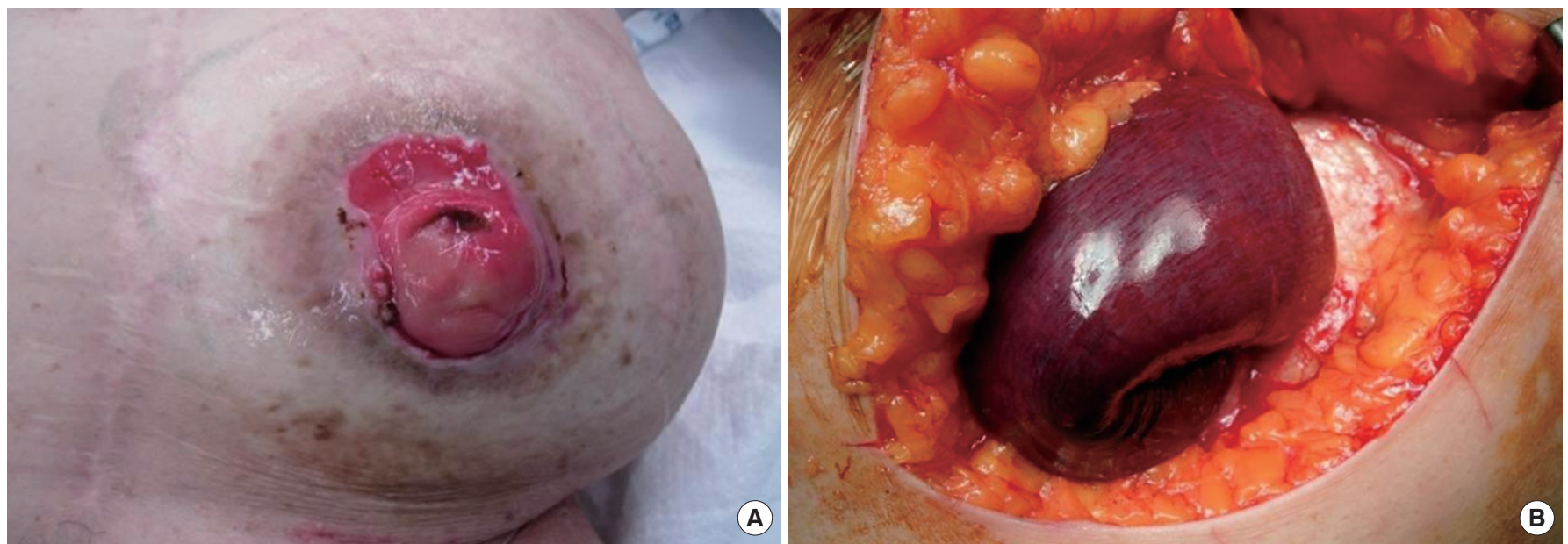

Fig. 4. Strangulated parastomal hernia. (A) External appearance of the parastomal hernia. Protrusion of the abdominal wall around the stoma opening. (B) Strangulated small bowel in a parastomal hernia.
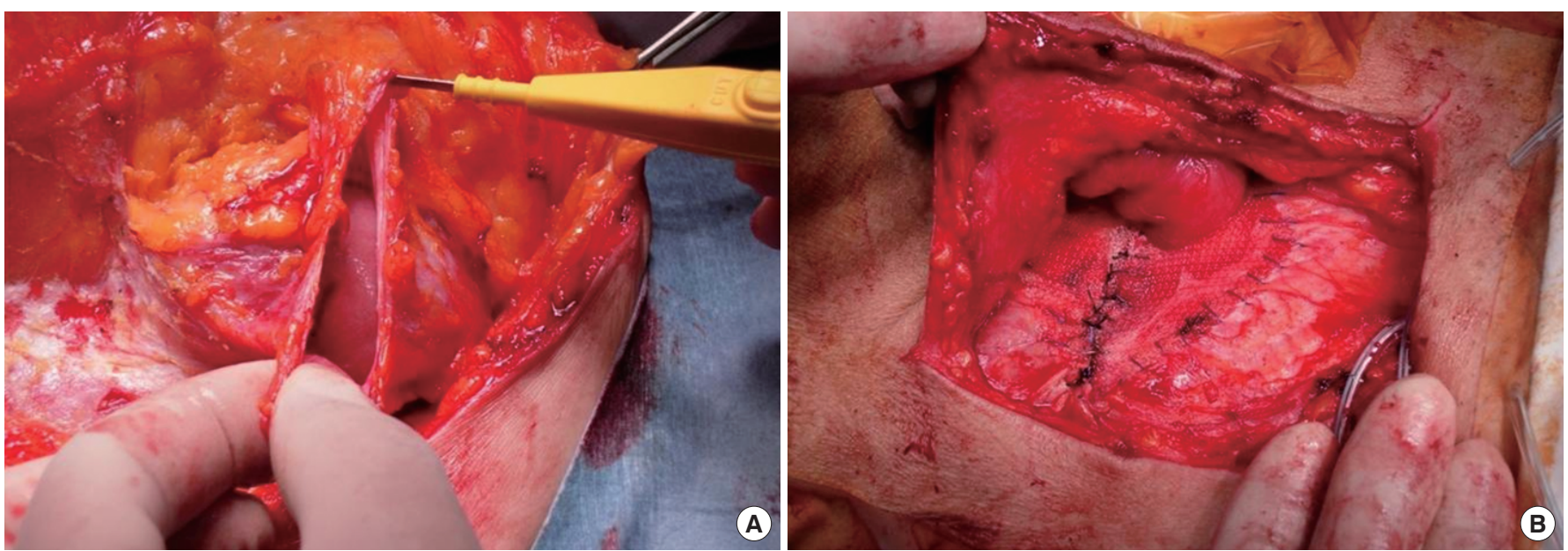

Fig. 5. Mesh repair of a parastoma hernia. (A) The hernia sac is opened during the operation to examine the herniated bowel and to facilitate reduction to the peritoneal cavity. (B) After repair of the fascia, mesh was overlaid to reinforce the abdominal wall around stoma.

\section{Results of emergency surgery}

Of the 39 patients, 4 patients underwent emergency surgery due to perforation of the herniated intestine (2 cases), mechanical obstruction (1 case) and strangulation (1 case) (Fig. 4). Three of the four patients had postoperative complications (Table 5). A 74-year-old male patient who underwent emergency suture repair due to an incarcerated hernia suffered from postoperative pneumonia and died after 9 days. Also, a 66-year-old female patient who underwent an emergency reposition procedure due to perforation of the herniated intestine developed a wound infection, and another 86-year-old male patient who underwent an emergency reposition procedure due to colon perforation by herniation had multiple complications, including bleeding, acute renal failure, respiratory failure and wound infection (Table 3).

The complication rate for patients who underwent emergency surgery was $75 \%$ ( 3 out of 4 patients), which was higher than the complication rate for patients who underwent elective surgery $(20.0 \% ; 7 / 35 ; \mathrm{P}>0.05)$. Other than the 1 patient who died after 2 weeks due to pneumonia, the average follow-up period for the 3 emergent surgery patients was $35.6 \pm 27.6$ months (range, 4 to 55 months), and there were no recurrence of the hernia during this period.

\section{DISCUSSION}

A parastomal hernia is an incisional hernia of the abdominal wall in the site of a stoma [4]. The incidence of parastomal hernias is not yet known, but most reports say that it is over 30\% [5]; it was reported to be $33 \%$ in a prospective study of end colostomy patients [6]. During the period of this study, the incidence of parastomal 
hernias for the responsible surgeon was $6.6 \%$ (19 out of 290 cases): $14.0 \%$ ( 8 out of 57 cases) for the abdominoperineal resection; $6.4 \%$ (9 out of 141 cases) for the Hartmann's operation; and 2.2\% (2 out of 92 cases) for the loop colostomy. Although there have been numerous studies on and devices for parastomal hernias until now, complete prevention and treatment are not easy to attain.

The frequency of parastomal hernias can vary from report to report, and it can vary with the types and the forms of the stomas. These differences, besides the different incidences themselves, can be attributed to the methods and the criteria used for diagnosis. A parastomal hernia is suspected in patients with subjective symptoms, and it can be diagnosed by physical examination with stoma pouches removed or by radiologic imaging. If the hernia reduces with ease when the patient is in the supine position due to the large defect in the abdominal wall or if the abdominal wall is too fat, a parastomal hernia may be difficult to diagnose through the physician's examination alone. Gurmu et al. [7] stated that diagnosis of a hernia might depend on the physician and that the result of the physician's examination might be different from that of radiologic imaging. A CT scan may not detect a hernia when the patient is in a supine position. As a solution to this, Janes et al. [8] mentioned that during a CT scan, the prone position of the patients can help the diagnosis of a parastomal hernia by easily evoking the hernia.

Types of stomas are also a factor in the incidence of parastomal hernias. In cases of end colostomies, incidences of $4-48 \%$ are being reported, and in cases of loop colostomies, $0-30.8 \%$ are being reported; in the case of the loop colostomy, the low incidence of parastomal hernias is due to its temporary nature, the follow-ups being short and the stoma being taken down before the hernia develops [4]. This is consistent with the fact that most of the patients in our study were end colostomies with permanent usage. Additionally, in the case of the end colostomy, the mesentery, including feeding vessels, must be a part of the anchoring suture to the abdominal wall. However, in the case of the loop colostomy, most of the part anchored to the abdominal wall is the anti-mesenteric border, which is easy to handle and so flexible that it can be readily anchored to the abdominal wall and can be adaptable to the muscle extension. The lower incidence of parastomal hernias of loop stomas can be attributed to these factors.

The causes of parastomal hernias are divided into patient factors and technical factors. Obesity, malnutrition, increased intra-abdominal pressure, steroids, malignancy, advanced age, and wound infection are referred to as patient factors, but with little evidence [4]. Meanwhile, Pilgrim et al. [6] reported in their recently conducted prospective studies that the size of the defect in the abdominal wall and the patient's age were independent contributing factors for parastomal hernias. Parastomal hernias developed in the early years, as in our patients, with the latest development reported being as late as 20 years [1] and half of the parastomal hernias developing in the first 2 years [4]. The clustered occurrence of parastomal hernias in the early years may support the importance of technical factors for the development of parastomal hernias.
The treatments for parastomal hernias have traditionally been relocation or suture repair of the fascia [9]. Although the relocation, contrary to our results, has been reported often as having better short-term results $[3,9]$, those studies were not prospectively randomized, so it is rash to jump to a conclusions. Since our study was not a prospective study either, the lower (and comparable to mesh repair) incidence of recurrence in suture repair patients than in relocation patients may be caused by fewer patients of large defects with severe hernias in the suture repair group. The only thing to note is that in the early study period, many reposition surgeries were performed, resulting in many recurrences; after the mesh repair procedure was started, recurrences were fewer (Fig. 5). Although the two surgical methods were not assigned in prospective random fashion, they were separated according to the timing (Fig. 2), and the suspicion that the patients expected to have low recurrence was artificially incorporated into mesh repair group, can be avoided which supports the reliability of this study. Even though the small number of enrolled patients prevents this result from being statistically significant, it result is consistent with that of other investigators. Now, mesh repair is regarded as the best surgical method for repairing a parastomal hernia [10]. We need further verification of the results with more patients in the future.

There were no cases of emergency surgery in the mesh repair group because perforation or strangulation of the herniated bowel may cause infection of the mesh, which is a very serious complication. The infection rates reported for non-absorbable mesh are not high (4-6\%), but these rates are for elective surgery [11, 12]. Mesh can be used safely in clean-contaminated surgery [13], but emergency surgery for such as bowel perforations may cause high risk due to contamination with stools or intestinal contents [10]. Infection or adhesion of the bowel on the mesh applied for the prevention or treatment of a parastomal hernia sometimes causes serious complications, and these are the most important reasons for the hesitation in using mesh in parastomal hernia operations [14].

In this study, surgical complications were observed in 3 out of 4 emergency surgery cases (75\%), and in 7 out of 35 (20\%) elective surgery cases. Among them, one patient who underwent emergency surgery died due to postoperative pneumonia. Emergency surgery is necessary in cases of incarceration, strangulation and possibility of perforation, and the complication rate becomes high in these situations. The larger number of complications in relocation patients is thought to be partly due to the larger number of emergency operations. Excluding the emergency operations, the complication rate for relocation patients of $16.7 \%$ ( 1 of 6 cases) is comparable to that of suture repair (25\%, 3 of 12 patients) and to that of mesh repair (17.6\%, 3 of 17 cases). Emergency surgery is not thought to elevate the recurrence rate because for each operation method, there were no recurrences in emergency surgery patients. However, because mesh cannot be used in emergency surgery due to the chance of infection, more recurrences may be associated with the operation method rather than the emergency situation per se, which can be addressed with more patients. Also, most 
results, including our results, were not based on prospective randomized studies, which implies that more patients with good general conditions were in the mesh repair group than in the relocation group. However, the fact that more recurrences did not occur in emergency operations means, paradoxically, that we must not say that the relocation method, which was performed more in patients with poor general condition, has a higher recurrence rate because it was performed on patients with poor general conditions.

The recent focus of parastomal hernias is on the preventive use of meshes and on the development of less-complicated biological materials. Serra-Aracil et al. [14] mentioned that preventive reinforcement of the fascia by using artificial materials during the initial stoma formation does not make complications higher with prevention of parastomal hernias. Janes et al. [15] stated, based on the result of a 5-year follow-up of randomized patients, that the superiority of preventive mesh lasted for over 5 years and that neither strictures nor fistulas were associated with the use of mesh. Additionally, Hammond et al. [16] reported lower complication rates when using collagen implants, which have higher biocompatibility than polypropylene mesh. We hope safer, more concrete, and more convenient methods for preventing parastomal hernias will be devised in the future.

Although we could not attain statistical significance due to the limited number of cases, mesh repair showed lower recurrence and complication rates and shorter hospital stays than relocation among the methods used for parastomal hernia surgery, and these results are consistent with those of others. Prospective randomized studies are necessary if more reliable evidence is to be obtained, especially, for complicated parastomal hernias, such as perforations or strangulations; even more research is needed.

\section{CONFLICT OF INTEREST}

No potential conflict of interest relevant to this article was reported.

\section{REFERENCES}

1. Leong AP, Londono-Schimmer EE, Phillips RK. Life-table analysis of stomal complications following ileostomy. Br J Surg 1994;81: 727-9.

2. Londono-Schimmer EE, Leong AP, Phillips RK. Life table analy- sis of stomal complications following colostomy. Dis Colon Rectum 1994;37:916-20.

3. Tekkis PP, Kocher HM, Payne JG. Parastomal hernia repair: modified thorlakson technique, reinforced by polypropylene mesh. Dis Colon Rectum 1999;42:1505-8.

4. Carne PW, Robertson GM, Frizelle FA. Parastomal hernia. Br J Surg 2003;90:784-93.

5. Israelsson LA. Preventing and treating parastomal hernia. World J Surg 2005;29:1086-9.

6. Pilgrim $\mathrm{CH}$, McIntyre R, Bailey M. Prospective audit of parastomal hernia: prevalence and associated comorbidities. Dis Colon Rectum 2010;53:71-6.

7. Gurmu A, Matthiessen P, Nilsson S, Pahlman L, Rutegard J, Gunnarsson U. The inter-observer reliability is very low at clinical examination of parastomal hernia. Int J Colorectal Dis 2011;26:89-95.

8. Janes A, Weisby L, Israelsson LA. Parastomal hernia: clinical and radiological definitions. Hernia 2011;15:189-92.

9. Riansuwan W, Hull TL, Millan MM, Hammel JP. Surgery of recurrent parastomal hernia: direct repair or relocation? Colorectal Dis 2010;12:681-6.

10. Luning TH, Spillenaar-Bilgen EJ. Parastomal hernia: complications of extra-peritoneal onlay mesh placement. Hernia 2009;13:487-90.

11. Geisler DJ, Reilly JC, Vaughan SG, Glennon EJ, Kondylis PD. Safety and outcome of use of nonabsorbable mesh for repair of fascial defects in the presence of open bowel. Dis Colon Rectum 2003;46: 1118-23.

12. Steele SR, Lee P, Martin MJ, Mullenix PS, Sullivan ES. Is parastomal hernia repair with polypropylene mesh safe? Am J Surg 2003; 185:436-40.

13. Schreinemacher MH, Beets GL, Bouvy ND. Prevention of parastomal hernia. It is possible! Ann Surg 2010;252:707-8.

14. Serra-Aracil X, Bombardo-Junca J, Moreno-Matias J, Darnell A, Mora-Lopez L, Alcantara-Moral M, et al. Randomized, controlled, prospective trial of the use of a mesh to prevent parastomal hernia. Ann Surg 2009;249:583-7.

15. Janes A, Cengiz Y, Israelsson LA. Preventing parastomal hernia with a prosthetic mesh: a 5-year follow-up of a randomized study. World J Surg 2009;33:118-21.

16. Hammond TM, Huang A, Prosser K, Frye JN, Williams NS. Parastomal hernia prevention using a novel collagen implant: a randomised controlled phase 1 study. Hernia 2008;12:475-81. 